\title{
Nonlinear Dynamics on the Plane AND INTEGRABLE HiERARCHIES OF INFINITESIMAL DEFORMATIONS
}

\author{
B. Konopelchenko*and L. Martínez Alonso ${ }^{\dagger}$ \\ Isaac Newton Institute for Mathematical Sciences, \\ University of Cambridge, 20 Clarkson Road, \\ Cambridge, CBз OEH, United Kingdom
}

\begin{abstract}
A class of nonlinear problems on the plane, described by nonlinear inhomogeneous $\bar{\partial}$-equations, is considered. It is shown that the corresponding dynamics, generated by deformations of inhomogeneous terms (sources) is described by Hamilton-Jacobi type equations associated with hierarchies of dispersionless integrable systems. These hierarchies are constructed by applying the quasiclassical $\bar{\partial}$-dressing method.
\end{abstract}

Key words: $\bar{\partial}$-method. Dispersionless hierarchies. 1991 MSC: 58B20.

*Permanent address: Dipartimento di Fisica, Universita di Lecce and Sezione INFN, 73100 Lecce, Italy. B. Konopelchenko is supported in part by COFIN 2000 "Sintesi".

${ }^{\dagger} \mathrm{L}$. Martinez Alonso is on leave of absence from Departamento de Fisica Teorica II, Universidad Complutense, E-28040 Madrid, Spain and is supported by the Fundacion Banco Bilbao Vizcaya Argentaria 


\section{Introduction}

Dispersionless integrable equations and hierarchies represent a particular class of integrable systems with a number of peculiar and remarkable properties [1]-[13]. They arise in various problems of physics and mathematics from hydrodynamics and quantum field models to the theory of conformal mappings (see e.g. [9], [14]-18]).

Recently it was shown [19]-21] that the dispersionless integrable hierarchies are amenable to the quasi-classical $\bar{\partial}$-dressing method and that they are closely connected with the theory of quasiconformal mappings on the plane [22]-24]. In these papers it was demonstrated that dispersionless integrable hierarchies are associated with the simple nonlinear $\bar{\partial}$-equation $S_{\bar{z}}=W\left(z, \bar{z}, S_{z}\right)$.

In the present paper we place this observation in a much wider setting. Namely, we consider a class of nonlinear problems on the plane which can be described by equations of the type

$$
S_{\bar{z}}=W\left(z, \bar{z}, S_{z}\right)+h(z, \bar{z})
$$

where $z, \bar{z} \in \mathbb{C}$ and $S, h$ and $W$ are complex-valued functions. Such equations arise in several problems of hydrodynamics, electrostatics and quasiconformal mappings. We assume that nonlinearity $W$ and source $h$ are separated. We are looking for solutions of (11) in the form $S=S_{0}+\widetilde{S}$, where $S_{0}$ is determined by the source $h$. In this way the construction of solutions $S$ of the problem (11) is nothing but the dressing of the background solution $S_{0}$ by the use of the quasi-classical $\bar{\partial}$-dressing method.

Here we will concentrate on the study of properties of infinitesimal deformations for the problem (1). We will show that these infinitesimal de-

formations $\left(\frac{\partial S}{\partial t_{n}}\right)$ obey universal (independent of the form of $W$ ) hierarchies of Hamilton-Jacobi (H-J) type equations, which in turn give rise to associated hierarchies of dispersionless integrable systems. To derive these systems we use the quasi-classical $\bar{\partial}$-dressing method. Particular parametrizations of variations of sources $h$ lead to different hierarchies of H-J equations and their associated dispersionless systems. Several concrete examples, like the dispersionless Kadomtsev-Petviashvili (KP) and the two-dimensional Toda lattice (2DTL) among others relevant examples, are considered. Equations arising in different gauges are also discussed. 


\section{General problem, parametrization of sources and the quasi-classical $\bar{\partial}$-dressing method}

So we will consider abstract nonlinear systems on the complex plane $\mathbb{C}$ described by an equation of the form

$$
S_{\bar{z}}=W\left(z, \bar{z}, S_{z}\right)+h(z, \bar{z}) .
$$

The function $W$ which defines the nonlinearity is assumed to be an analytic function of $S_{z}$ (i.e. independent of $\bar{S}_{z}$ ). The inhomogeneous term $h$ can be treated as an external source for the nonlinear system.

Equations of the form (2) arise in different fields of physics and mathematics. For example:

1. Several problems of the plane motion of fluids [25], generalized growth and Hele-Shaw problems [26].

2. After differentiation with respect to $z$, equation (2) reads

$$
S_{z \bar{z}}=\left(W\left(z, \bar{z}, S_{z}\right)\right)_{z}+\rho(z, \bar{z})
$$

where $\rho \equiv h_{z}$, so that it can be treated as the nonlinear Poisson equation for the potential $S$. Such equation may arise in some special types of effective potential models in which $W_{z}$ and $\rho$ describe an effective nonlinearity and a given external source, respectively.

3. Under certain conditions solutions of (2) define quasiconformal mappings of plane domains (see e.g. [27]-[28]). Equations of the type (2)arise also in the study of extremal problems for quasiconformal mappings (see [29).

Thus, the results obtained for equation (2) may have a wide range of applications.

In our discussion we will consider the class of nonlinear problems (2) in which nonlinearity and sources are separated, i.e. we assume that there is a partition of the complex plane

$$
\mathbb{C}=G_{0} \bigcup \widetilde{G}, \quad G_{0} \bigcap \widetilde{G}=\varnothing
$$


such that

$$
\begin{aligned}
& h(z, \bar{z})=0, \quad z \in G_{0} \\
& W\left(z, \bar{z}, S_{z}\right)=0, \quad z \in \widetilde{G} .
\end{aligned}
$$

We will look for solutions of equation (2) of the form

$$
S=S_{0}+\widetilde{S}
$$

where $\widetilde{S}$ is bounded on $\mathbb{C}$ and

$$
\begin{aligned}
& S_{0, \bar{z}}=0, \quad z \in G_{0} \\
& \widetilde{S}_{\bar{z}}=0, \quad z \in \widetilde{G} .
\end{aligned}
$$

The representation ([6), (7) imposes no constraint on the solution of (2). In virtue of (5)-(7), equation (2) is equivalent to the system

$$
\begin{aligned}
& S_{0, \bar{z}}=h, \quad z \in \mathbb{C} \\
& \widetilde{S}_{\bar{z}}=W\left(z, \bar{z}, S_{0, z}+\widetilde{S}_{z}\right), \quad z \in \mathbb{C} .
\end{aligned}
$$

Thus, $S_{0}$ is determined by the source $h$ and can be considered as a background (seed) solution of (2) corresponding to $W \equiv 0$. Hence, the whole procedure of construction of solutions of (目) (or the equivalent system (8)) is the dressing method, a well-known procedure in the theory of integrable equations (see e.g. [30]-[32]). A peculiar feature of the dressing method for (2) is that the corresponding $\bar{\partial}$-equation is a local nonlinear partial differential equation. This $\bar{\partial}$-equation can be considered as the quasi-classical limit of the standard nonlocal $\bar{\partial}$-equation [19]-21]. The method of construction of solutions within this quasiclassical $\bar{\partial}$-dressing method [19-21] consists, basically, in solving (8) or the quasi-linear equation for $m \equiv S_{z}$ in the domain $G_{0}$ and the gluing with $S$ in the domain $\widetilde{G} 20$.

In this paper we will concentrate on the study of the properties of deformations of solutions of equation (2). Infinitesimal deformations (variations) $S \rightarrow S+\delta S$, generated by infinitesimal deformations $h \rightarrow h+\delta h$ of the source, are determined by the linear inhomogeneous Beltrami equation

$$
(\delta S)_{\bar{z}}=W^{\prime}\left(z, \bar{z}, S_{z}\right)(\delta S)_{z}+\delta h,
$$


where $W^{\prime}(z, \bar{z}, \xi) \equiv \frac{\partial W(z, \bar{z}, \xi)}{\partial \xi}$. Properties of the Beltrami equation are wellstudied (see e.g. [33, [22], 23]). For our analysis we need two of them [33]:

1. If $f_{1}, \ldots, f_{n}$ are solutions of the homogeneous Beltrami equation $f_{\bar{z}}=$ $\mu f_{z}$, then any arbitrary differentiable function $F\left(f_{1}, \ldots, f_{n}\right)$ is a solution too

2. If the function $\mu$ satisfies $|\mu| \leq k<1$, then the only solution $f_{\bar{z}}=\mu f_{z}$ such that $f_{\bar{z}}$ is locally $L^{p}$ for some $p>2$, and such that $f$ vanishes at some point of the extended plane $\mathbb{C}^{*}$ is $f \equiv 0$.

These two properties provide a basis for the quasiclassical $\bar{\partial}$-dressing method [19], [21]. The first one implies that, together with the infinitesimal deformations $\delta_{1} S, \delta_{2} S, \ldots, \delta_{n} S$, any differentiable function of them

$F\left(\delta_{1} S, \delta_{2} S, \ldots, \delta_{n} S\right)$ is also a solution of the Beltrami equation $f_{\bar{z}}=W^{\prime} f_{z}$ in the domain $G_{0}$. Furthermore, under the conditions of the second property, if one is able to find a function $F$ such that $F\left(\delta_{1} S, \delta_{2} S, \ldots, \delta_{n} S\right)$ is bounded on $\mathbb{C}$ and vanishes at some point of $\mathbb{C}^{*}$, one has

$$
F\left(\delta_{1} S, \delta_{2} S, \ldots, \delta_{n} S\right)=0 .
$$

The derivation of equations of the type (10) is one of the main goals of the quasiclassical $\bar{\partial}$-dressing method. In this paper we aim to determine these kind of relations for the infinitesimal deformations of the problem (2). To do that one has to parametrize the source $h$ and its deformations in one or another way.

Having in mind the relation $S_{0, z \bar{z}}=h_{z}=\rho(z, \bar{z})$, it is quite natural to choose $\rho$ in the form

$$
\rho=h_{z}=\sum_{\alpha=1}^{N} \sum_{n=0}^{\infty} \gamma_{\alpha n} \delta^{(n, 0)}\left(z-z_{\alpha}\right),
$$

where $\gamma_{\alpha n}$ and $z_{\alpha}$ are arbitrary complex constants $\left(z_{\alpha} \in \widetilde{G}\right)$ and $\delta^{(n, 0)}(\xi)$ are the $z$-derivatives of Dirac delta functions. Since

$$
S_{0}(z, \bar{z})=\iint_{\widetilde{G}} d z^{\prime} \wedge d \bar{z}^{\prime} \ln \left(z-z^{\prime}\right) \rho\left(z^{\prime}, \bar{z}^{\prime}\right)
$$

one has

$$
S_{0}(z, \bar{z})=\sum_{\alpha=1}^{N}\left(t_{0 \alpha} \ln \left(z-z_{\alpha}\right)+\sum_{n=1}^{\infty} \frac{t_{n \alpha}}{\left(z-z_{\alpha}\right)^{n}}\right)
$$


where $t_{0 \alpha}=\gamma_{\alpha 0}$ and $t_{n \alpha}=(-1)^{n}(n-1) ! \gamma_{n \alpha}, \quad n \geq 1$.

Of course, all pole-type singularities in (12) can be obtained by coalescing logarithmic terms, so that only the $\delta^{(n, 0)}$ terms in (11) and the $t_{0 \alpha} \ln \left(z-z_{\alpha}\right)$ terms in (12) are, in fact, of fundamental importance. But it is convenient to add other terms to the source $h$ from the very beginning instead of performing coalescence at the end.

By considering an infinite number of points $z_{\alpha}$ and by passing from the sum in (11) to an integral, one gets a source $\rho$ of generic form. However, the simpler forms (11) and (12) are much more convenient for performing calculations. The symbols $t_{n \alpha}$ and $z_{\alpha}(\alpha=1, \ldots, N ; n \geq 0)$ are free parameters, so that within the class of sources given by (11) the deformations (variations) of the sources are generated by variations of $t_{n \alpha}$ and $z_{\alpha}$. In this case $\delta h=\epsilon \frac{\partial h}{\partial \tau}$ and $\delta S=\epsilon \frac{\partial S}{\partial \tau}$, where $\epsilon$ is an infinitesimal parameter and $\tau$ is any of the parameters $t_{n \alpha}$ and $z_{\alpha}$. Hence, we have

$$
\left(S_{\tau}\right)_{\bar{z}}=W^{\prime} \cdot\left(S_{\tau}\right)_{z}+h_{\tau}
$$

In virtue of (6) and (12) one has

$$
\begin{aligned}
& S_{t_{0 \alpha}}=\ln \left(z-z_{\alpha}\right)+\widetilde{S}_{t_{0 \alpha}}, \\
& S_{t_{n \alpha}}=\frac{1}{z-z_{\alpha}}+\widetilde{S}_{t_{n \alpha}},
\end{aligned}
$$

and

$$
S_{z_{\alpha}}=-\frac{t_{0 \alpha}}{z-z_{\alpha}}+\sum_{n \geq 1} \frac{n t_{n \alpha}}{\left(z-z_{\alpha}\right)^{n+1}}+\widetilde{S}_{z_{\alpha}} .
$$

These expressions have singularities, but all of them are located in the domain $\widetilde{G}$.

Now let us consider a differentiable function of the type $F\left(S_{t_{n \alpha}}, S_{z_{\alpha}}, \ldots\right)$. It is a regular function on the domain $G_{0}$. Hence due to the first property of the Beltrami equation, one has

$$
F_{\bar{z}}=W^{\prime} F_{z}, \quad z \in G_{0} .
$$

In general this function has singularities in the domain $\widetilde{G}$ due to the singular terms of $S_{0 \tau}$. But if we manage to cancel these singularities by a right choice of $F$, then we have

$$
F_{\bar{z}}=0, \quad z \in \widetilde{G},
$$


so that

$$
F_{\bar{z}}=W^{\prime} F_{z}, \quad z \in \mathbb{C} .
$$

In this case, as we are assuming that $\widetilde{S}$ is bounded in $\mathbb{C}$, if $F\left(S_{t_{n \alpha}}, S_{z_{\alpha}}, \ldots\right)$ vanish at some point of the extended complex plane then from the second basic property of Beltrami equation we conclude

$$
F\left(S_{t_{n \alpha}}, S_{z_{\alpha}}, \ldots\right)=0 .
$$

Equations like (19) are our desired equations relating infinitesimal symmetries of the system (2). The next sections of the present paper are devoted to the derivation of hierarchies of equations of this type. As we shall see the form of the equations (19) does not depend on the choice of $W$, so that they have a universal character.

The problem (21) and the equations (19) have an additional important property; namely, they are invariant under the gauge transformations

$$
S(z, \bar{z}, \tau) \rightarrow S^{\prime}(z, \bar{z}, \tau)=S(z, \bar{z}, \tau)+g(\tau),
$$

where $g(\tau)$ is an arbitrary function. For concrete equations this property has been observed earlier in [10]. This invariance implies that we can arbitrarily prescribe the value of $S$ at any given point $z_{0}$ (except at one of the $z_{\alpha}$ ), i.e. we can always normalize $S$ by the condition $S\left(z_{0}, \tau\right)=g_{0}\left(t_{n \alpha}, z_{\alpha}\right)$, in particular $S\left(z_{0}\right)=0$. At the point $z=z_{\alpha}$, one can choose the gauge $\widetilde{S}\left(z_{\alpha}\right)=0$. As we shall see different gauges produce different, but related, equations of the type (19). It is also possible to give a gauge invariant formulation of equations (19).

Before proceeding to the construction of concrete equations we would like to note the following:

Remark 2.1 Equation (3) is the Euler-Lagrange equation with the Lagrangian

$$
L=\frac{1}{2} S_{z} S_{\bar{z}}-\Phi\left(z, \bar{z}, S_{z}\right)+\rho S
$$

where $\frac{\partial \Phi\left(z, \bar{z}, S_{z}\right)}{\partial\left(S_{z}\right)}=W\left(z, \bar{z}, S_{z}\right)$. Equation (3) can be also represented in the form of the conservation law 


$$
\left(\frac{1}{2} S_{z}\right)_{\bar{z}}+\left(\frac{1}{2} S_{\bar{z}}-W\left(z, \bar{z}, S_{z}\right)-h\right)_{z}=0
$$

Formally this conservation law is connected with the gauge invariance mentioned above.

Remark 2.2 It is well-known that for the plane potential flows the kinetic energy of a fluid coincides with the Dirichlet integral (see e.g. [34]). In our case the total "kinetic energy" $\frac{1}{2} \iint_{\mathbb{C}} S_{z} S_{\bar{z}} d z \wedge d \bar{z}$ diverges due to singularity of $S_{0}$. A regularized "kinetic energy" can be defined as the complex Dirichlet type integral

$$
D=\frac{1}{2} \iint_{\mathbb{C}}\left(\partial S \wedge \bar{\partial} S-\partial S_{0} \wedge \bar{\partial} S_{0}\right)=\frac{1}{4 i} \iint_{\mathbb{C}}\left(d S \wedge * d S-d S_{0} \wedge * d S_{0}\right) .
$$

Remark 2.3 Within the fluid mechanical interpretation of the problem (2) the $\gamma_{0 \alpha} \delta^{(0,0)}\left(z-z_{\alpha}\right)$ terms in the source $\rho$ or the logarithmic terms $t_{0 \alpha} \ln \left(z-z_{\alpha}\right)$ describe sources or sinks of the fluid, while other terms describe vortices located at the points $z_{\alpha}$ (see e.g. [34]). In the electrostatic applications the $\gamma_{0 \alpha} \delta^{(0,0)}\left(z-z_{\alpha}\right)$ terms or $t_{0 \alpha} \ln \left(z-z_{\alpha}\right)$ terms, obviously correspond to point charged particles with charges $t_{0 \alpha}$. Other terms describe contributions from electrical multipoles. Thus, the deformations we are discussing here are generated by the infinitesimal variations of the strengths and positions of sources or sinks of fluid, charges of the point particles and their positions, and strengths and positions of vortices and multipoles.

Remark 2.4 Equations of the type (19) can be treated as the dispersionless limit of certain dispersive integrable hierarchies. Under this limit wave functions $\psi$ of the fully dispersive case become $\psi=\exp \left(\frac{S}{\epsilon}\right)$ where $\epsilon \rightarrow 0$. Within such a connection the variables $t_{n \alpha}$ represent slow variables associated with the standard flow variables $x_{n \alpha}=\frac{t_{n \alpha}}{\epsilon}$. The variables $t_{0 \alpha}$ can be considered as a quasi-classical limit of the Miwa variables. Indeed, in terms of Miwa variables, the undressed dispersive wave function $\psi_{0}$ has the form $\psi_{0}(z)=\prod_{\alpha=1}^{N}\left(\frac{z}{z_{\alpha}}-1\right)^{p_{\alpha}}$ where $p_{\alpha}$ are integers (see [35]). By considering large $p_{\alpha}$ and by introducing $t_{0 \alpha}$ via $p_{\alpha}=t_{0 \alpha} / \epsilon(\epsilon \rightarrow 0)$, one gets

$$
\psi_{0} \sim \exp \frac{\sum_{\alpha=1}^{N} t_{0 \alpha} \ln \left(z-z_{\alpha}\right)}{\epsilon} \sim \exp \left(\frac{S_{0}}{\epsilon}\right)
$$




\section{One point-like source case}

We begin by the simplest case of source $\rho$ concentrated on a single point, i.e.

$$
S_{0}=t_{0} \ln \left(z-z_{\alpha}\right)+\sum_{n=1}^{\infty} \frac{t_{n}}{\left(z-z_{\alpha}\right)^{n}} .
$$

Therefore

$$
\begin{aligned}
& \frac{\partial S}{\partial t_{0}}=\ln \left(z-z_{\alpha}\right)+\frac{\partial \widetilde{S}}{\partial t_{0}}, \\
& \frac{\partial S}{\partial t_{n}}=\frac{1}{\left(z-z_{\alpha}\right)^{n}}+\frac{\partial \widetilde{S}}{\partial t_{n}} .
\end{aligned}
$$

From these equations we deduce

$$
e^{-\frac{\partial S}{\partial t_{0}}}=\frac{1}{z-z_{\alpha}} e^{-\frac{\partial \widetilde{S}}{\partial t_{0}}}, \quad z \in \mathbb{C}
$$

and

$$
e^{-\mathcal{D}\left(z_{1}\right) S(z)}=\frac{z-z_{1}}{z-z_{\alpha}} e^{-\mathcal{D}\left(z_{1}\right) \widetilde{S}(z)}, \quad z \in \mathbb{C}, z_{1} \in \widetilde{G},
$$

where $\mathcal{D}(z)$ stands for the quasiclassical vertex operator

$$
\mathcal{D}(z):=\sum_{n=1}^{\infty} \frac{1}{n}\left(z-z_{\alpha}\right)^{n} \frac{\partial}{\partial t_{n}} .
$$

Let us first consider variations of the the $t_{n}$ variables (time variables). By

counting singularities of the derivatives $\frac{\partial S}{\partial t_{n}}$, one concludes that the simplest of the equations of the type (14) is of the form

$$
\begin{aligned}
& S_{t_{2}}-\left(S_{t_{1}}\right)^{2}-v S_{t_{1}}-u=0, \\
& v=-2 \widetilde{S}_{t_{1}}\left(z_{\alpha}\right), \quad u=S_{t_{2}}\left(z_{0}\right)-\left(S_{t_{1}}\left(z_{0}\right)\right)^{2}-v S_{t_{1}}\left(z_{0}\right),
\end{aligned}
$$

where $z_{0}$ is an arbitrary point $z_{0} \neq z_{\alpha}$. Other analogous equations look like

$$
S_{t_{n}}-\left(S_{t_{1}}\right)^{n}-\sum_{k \geq 0}^{n-1} v\left(S_{t_{1}}\right)^{k}=0, \quad n \geq 2
$$


where the functions $v_{k}=v_{k}(t)$ are appropriate combinations of derivatives of $\widetilde{S}\left(z_{\alpha}\right)$ and $S\left(z_{0}\right)$ with respect to $t_{1}$.

Equations (26) form a complete set of equations for infinitesimal variations $S_{t_{n}}$ of $S$, generated by variations of the times $t_{n}$. They are equations of Hamilton-Jacobi type.

An important property of equations (26) is that they have the same form for all functions $W\left(z, \bar{z}, S_{z}\right)$ describing nonlinearities in the problems (2).

Remark 3.1 Within the quasi-classical $\bar{\partial}$-dressing method, the functions $W$ play a role of quasi-classical $\bar{\partial}$-data [19], [21]. The independence of the form of integrable equations on the $\bar{\partial}$-data is a general feature of the dressing method.

By construction, all the equations (26) are compatible and solvable by the use of the $\bar{\partial}$-problem (2) and, in turn, they determine a hierarchy of equations for the functions $u, v$ and so on. The form of these equations depends on the gauge choice.

There are two natural gauges. The first one is $S\left(z_{0}\right)=0,\left(z_{0} \neq z_{\alpha}\right)$, in which $u=0$ and the hierarchy (26) becomes the Hamilton-Jacobi system of equations for the dispersionless modified KP (dmKP) hierarchy. The lowest member of which is [36], [21]

$$
v_{t_{3}}+\frac{3}{2} v^{2} v_{t_{1}}-\frac{3}{4} v_{t_{1}} \partial_{t_{1}}^{-1}\left(v_{t_{2}}\right)-\frac{3}{4} \partial_{t_{1}}^{-1}\left(v_{t_{2} t_{2}}\right)=0
$$

The second natural gauge is $\widetilde{S}\left(z_{\alpha}\right)=0$. It leads to $v=0$, and then (26) reduce to the system of Hamilton-Jacobi equations for the well-known dKP hierarchy (see e.g. [1]-12]). The dKP equation itself is

$$
u_{t_{3}}=\frac{3}{2} u u_{t_{1}}+\frac{3}{4} \partial_{t_{1}}^{-1}\left(u_{t_{2} t_{2}}\right)
$$

Other gauges can be of interest too. For example, if we impose $S\left(z_{0}\right)=$ $\frac{t_{1}}{z_{0}-z_{\alpha}}$, i.e. $\widetilde{S}\left(z_{0}\right)=0$, we get a hierarchy of $2+1$-dimensional Gardner (mixed $\mathrm{KP}-\mathrm{mKP}$ ) equations. We shall refer to the hierarchy of equations (26) and associated hierarchy for $u$ and $v$ as the $\mathrm{d}(\mathrm{KP}-\mathrm{mKP})$ hierarchy.

One can formulate equations in gauge invariant form. In such a formulation one has equations (26) for the gauge invariant object $S^{*}:=S(z)-$ $S\left(z_{0}\right)$, while equations for potentials $v_{k}$ are formulated in terms of the gaugeinvariant

$$
w:=u+\frac{1}{2} \partial_{t}^{-1} v_{t_{2}}-\frac{1}{4} v^{2}
$$


This formulation provides us also with the dispersionless Miura transformation [36]

$$
u=\frac{1}{2} \partial_{t}^{-1} v_{t_{2}}-\frac{1}{4} v^{2}
$$

By proceeding along the same lines as in [37], one can derive several important generating equations and addition formulae associated with equations (26). In virtue of (23), both $p:=\frac{\partial S}{\partial t_{1}}$ and $\exp \left(-\mathcal{D}\left(z_{1}\right) S(z)\right)$ have a simple pole at $z=z_{\alpha}$, so that one gets

$$
p(z)-p\left(z_{0}\right)=-\frac{1}{z_{1}-z_{\alpha}}\left(e^{-\mathcal{D}\left(z_{1}\right) S(z)}-e^{-\mathcal{D}\left(z_{1}\right) S\left(z_{0}\right)}\right),
$$

where $z_{0} \in \mathbb{C}$ is an arbitrary point $z_{0} \neq z_{\alpha}$.

Equation (29) is of fundamental importance for describing infinitesimal deformations. It is the generating equation for the hierarchy of equations (26). Indeed, by expanding both sides of (29) in series on $z_{1}$, one gets a system which is equivalent to (26). Furthermore, by evaluating both sides of (29) at $z_{0}=z_{1}$, one gets

$$
p(z)-p\left(z_{1}\right)=-\frac{1}{z_{1}-z_{\alpha}} \exp \left(-\mathcal{D}\left(z_{1}\right)\left(S(z)-\widetilde{S}\left(z_{\alpha}\right)\right)\right), \quad z \in \mathbb{C}, z_{1} \in \widetilde{G} .
$$

Thus, by setting $z=z_{2} \in \widetilde{G}$ and using (23) and the skew-symmetry of the left-hand side of (30) under the interchange $z_{1} \leftrightarrow z_{2}$, one concludes that

$$
\mathcal{D}\left(z_{1}\right)\left(\widetilde{S}\left(z_{2}\right)-\widetilde{S}\left(z_{\alpha}\right)\right)=\mathcal{D}\left(z_{2}\right)\left(\widetilde{S}\left(z_{1}\right)-\widetilde{S}\left(z_{\alpha}\right)\right) .
$$

Equation (31) implies that there exists a function $F$ such that

$$
\widetilde{S}(z)=\widetilde{S}\left(z_{\alpha}\right)-\mathcal{D}(z) F .
$$

So one has

$$
p\left(z_{2}\right)-p\left(z_{1}\right)=\frac{\left(z_{2}-z_{1}\right)}{\left(z_{\alpha}-z_{1}\right)\left(z_{2}-z_{\alpha}\right)} e^{\mathcal{D}\left(z_{1}\right) \mathcal{D}\left(z_{2}\right) F}, \quad z_{1}, z_{2} \in \widehat{G} .
$$

Further, by summing up the relation (33) for pairs of points $\left(z_{2}, z_{1}\right),\left(z_{1}, z_{3}\right)$ and $\left(z_{3}, z_{2}\right)$, one gets

$$
\begin{gathered}
\left(z_{1}-z_{2}\right)\left(z_{3}-z_{\alpha}\right) e^{\mathcal{D}\left(z_{1}\right) \mathcal{D}\left(z_{2}\right) F}+\left(z_{3}-z_{1}\right)\left(z_{2}-z_{\alpha}\right) e^{\mathcal{D}\left(z_{3}\right) \mathcal{D}\left(z_{1}\right) F} \\
+\left(z_{2}-z_{3}\right)\left(z_{1}-z_{\alpha}\right) e^{\mathcal{D}\left(z_{2}\right) \mathcal{D}\left(z_{3}\right) F}=0, \quad z_{1}, z_{2}, z_{3} \in \widetilde{G}
\end{gathered}
$$


It is the addition formula for the dKP hierarchy in the case when the singularity is located at a finite point $z_{\alpha}$. One can get the usual form of the dKP addition formula (see e.g. [11], [12, [18]) by sending $z_{\alpha} \rightarrow \infty$. Equation (34), which is gauge invariant, means that $F=\ln \tau_{d K P}$, where $\tau_{d K P}$ stands for the tau-function of the dKP hierarchy [7], [8], [1], [12].

From (29) one finds also that

$$
\begin{gathered}
\ln \left[p(z)-p\left(z_{0}\right)\right]-\ln \left(z_{\alpha}-z_{i}\right)-\mathcal{D}\left(z_{i}\right) \widetilde{S}\left(z_{\alpha}\right) \\
=\ln \left[e^{-\mathcal{D}\left(z_{i}\right) S(z)}-e^{-\mathcal{D}\left(z_{i}\right) S\left(z_{0}\right)}\right], \quad z_{i} \in \widetilde{G},(i=1,2,3) .
\end{gathered}
$$

Acting on this equation by the operators $\mathcal{D}\left(z_{k}\right)$ and $\mathcal{D}\left(z_{l}\right)$ with different values of the indexes $k, l, i$, and summing up the corresponding equations, one gets

$$
\sum_{i, k, l} \epsilon_{i k l}\left(\mathcal{D}\left(z_{i}\right)-\mathcal{D}\left(z_{k}\right)\right) \ln \left[e^{-\mathcal{D}\left(z_{l}\right) S(z)}-e^{-\mathcal{D}\left(z_{l}\right) S\left(z_{0}\right)}\right]=0, \quad z \in \mathbb{C}, z_{i} \in \widetilde{G},
$$

where $\epsilon_{i k l}$ is the totally antisymmetric tensor $\left(\epsilon_{123}=1\right)$. Equation (36) is the generating equation for the hierarchy of equations for $S(z, \bar{z}, \boldsymbol{t})$. Indeed, the taylor expansion of its left-hand side leads to an infinite family of equations for $S$ only. For example, in the gauge $\widetilde{S}\left(z_{\alpha}\right)=0$, the first nontrivial order gives

$$
S_{t_{1} t_{3}}=\frac{3}{4} S_{t_{2} t_{2}}+\frac{3}{2}\left[S_{t_{2}}-\left(S_{t_{1}}\right)^{2}\right] S_{t_{1} t_{1}}
$$

that is the $S$-equation for the dKP equation [21]. In the gauge $S\left(z_{0}\right)=0$ $\left(z_{0} \neq z_{\alpha}\right)$, one has the corresponding equation for the dmKP hierarchy. Equation (36) encodes all relations between infinitesimal deformations generated by variations of times $t_{n}$.

Now let us include also into consideration the variations of strength of the logarithmic term in (20). Using (21) and (22), one readily gets

$$
\begin{aligned}
& S_{t_{1}}=A_{11} e^{-S_{t_{0}}}+A_{10}, \\
& S_{t_{2}}=A_{11}^{2} e^{-2 S_{t_{0}}}+A_{21} e^{-S_{t_{0}}}+A_{20},
\end{aligned}
$$


where

$$
\begin{aligned}
& A_{11}=e^{\widetilde{S}_{t_{0}}\left(z_{\alpha}\right)}, \quad A_{20}=S_{t_{2}}\left(z_{0}\right)-A_{11}^{2} e^{-2 S_{t_{0}}\left(z_{0}\right)}-A_{21} e^{-S_{t_{0}}\left(z_{0}\right)}, \\
& A_{21}=2 A_{11}\left(\frac{\partial \widetilde{S}_{t_{0}}(z)}{\partial z}\right)_{z=z_{\alpha}}, \quad A_{10}=S_{t_{1}}\left(z_{0}\right)-A_{11} e^{-S_{t_{0}}\left(z_{0}\right)} .
\end{aligned}
$$

In general,

$$
S_{t_{n}}=\sum_{k=0}^{n} A_{n k} e^{-k S_{t_{0}}}
$$

where $A_{n k}\left(t_{0}, \vec{t}\right)$ are certain functions.

The hierarchy of Hamilton-Jacobi equations (40) is a basic one for the one-point-like source (20). Equations (40) imply a hierarchy of integrable equations for the coefficients $A_{n k}$. The lowest member of this hierarchy arises from the system of equations (38) and in the gauge $S\left(z_{0}\right)=0$ looks like

$$
\begin{aligned}
& \phi_{t_{1}}+\psi_{t_{0}}-e^{\phi_{t_{0}}}=0, \\
& \phi_{t_{2}}+2 \psi_{t_{1}}+\left(\phi_{t_{1}}\right)^{2}+\left(\psi_{t_{0}}\right)^{2}=0,
\end{aligned}
$$

where $\phi:=\widetilde{S}\left(z_{\alpha}\right), \psi:=\widetilde{S}_{z}\left(z_{\alpha}\right)$.

Equation (28) allows us to derive an analog of the relationships (29)-(34) for the logarithmic times. Indeed, by substituting (38) into (29) one obtains

$$
\begin{gathered}
e^{-\mathcal{D} S(z)}-e^{-\mathcal{D} S\left(z_{0}\right)}+\frac{1}{z_{1}-z_{\alpha}} e^{\left(\mathcal{D}+\mathcal{D}\left(z_{1}\right)\right) S(z)} . \\
\cdot\left[e^{-\mathcal{D}\left(z_{1}\right) S(z)}-e^{-\mathcal{D}\left(z_{1}\right) S\left(z_{0}\right)}\right]=0,
\end{gathered}
$$

where we denote $\mathcal{D} \equiv \frac{\partial}{\partial t_{0}}$.

Similar to the case (29), equation (42) is the generating equation for the hierarchy of equations (40). They are obtained by the expansion of the lefthand side of (42) in Taylor series in $z_{1}$. Then, by evaluating (42) at $z_{0}=z_{1}$, one gets

$$
e^{-\mathcal{D}\left(S(z)-\widetilde{S}\left(z_{\alpha}\right)\right)}-e^{-\mathcal{D}\left(S\left(z_{1}\right)-\widetilde{S}\left(z_{\alpha}\right)\right)}+\frac{1}{z_{1}-z_{\alpha}} e^{-\mathcal{D}\left(z_{1}\right)\left(S(z)-\widetilde{S}\left(z_{\alpha}\right)\right)}=0,
$$


where $z \in \mathbb{C}, z_{1} \in \tilde{G}$, which implies (32). Evaluating (43) at $z=z_{2} \in \tilde{G}$ and using (23) and (32), one readily obtains the addition formulas for $F$ :

$$
\frac{1}{z_{2}-z_{\alpha}} e^{\mathcal{D D}\left(z_{2}\right) F}-\frac{1}{z_{1}-z_{\alpha}} e^{\mathcal{D D}\left(z_{1}\right) F}+\frac{z_{2}-z_{1}}{\left(z_{1}-z_{\alpha}\right)\left(z_{2}-z_{\alpha}\right)} e^{\mathcal{D}\left(z_{1}\right) \mathcal{D}\left(z_{2}\right) F}=0,
$$

where $z_{1}, z_{2} \in \tilde{G}$. Formulae (40), (41)-(44) incorporate those of the d(KPmKP) hierarchy. It is easy to see that (44) implies the dKP addition formula (34). It is straightforward to check that the function $S$, which obeys (40), solves also equations (26) and, in particular, equations (38) imply (25). Thus, it means that the logarithmic source is fundamental for the description of deformations of the problem (2).

Finally we consider variations of the positions $z_{\alpha}$ where the source $\rho$ is concentrated. For the pure logarithmic source $S_{0}=t_{0} \ln \left(z-z_{\alpha}\right)$ and

$$
\frac{\partial S}{\partial z_{\alpha}}=-\frac{t_{0}}{z-z_{\alpha}}+\frac{\partial \widetilde{S}}{\partial z_{\alpha}} .
$$

Using (22), one deduces that

$$
\frac{\partial S(z)}{\partial z_{\alpha}}+A e^{-S_{t_{0}}(z)}+B=0
$$

where $A=t_{0} \exp \widetilde{S}_{t_{0}}\left(z_{\alpha}\right)$ and $B=-\frac{\partial S}{\partial z_{\alpha}}\left(z_{0}\right)-A \exp \left(-S_{t_{0}}\left(z_{0}\right)\right), \quad\left(z_{0} \in \mathbb{C}, z_{0} \neq\right.$ $\left.z_{\alpha}\right)$.

In the generic case 20 )

$$
\frac{\partial S}{\partial z_{\alpha}}=-\frac{t_{0}}{z-z_{\alpha}}+\sum_{n \geq 1} \frac{n t_{n}}{\left(z-z_{\alpha}\right)^{n+1}}+\frac{\partial \widetilde{S}}{\partial z_{\alpha}} .
$$

In virtue of (21) one has

$$
\triangle S=-\frac{t_{0}}{z-z_{\alpha}}+\sum_{n \geq 1} \frac{n t_{n}}{\left(z-z_{\alpha}\right)^{n+1}}+\triangle \widetilde{S},
$$

where

$$
\triangle \equiv-t_{0} \frac{\partial}{\partial t_{1}}+\sum_{n \geq 1} n t_{n} \frac{\partial}{\partial t_{n+1}}
$$


Using (47) and (48), one concludes that

$$
\frac{\partial S}{\partial z_{\alpha}}-\triangle S+E=0
$$

where $E=-\frac{\partial S\left(z_{0}\right)}{\partial z_{\alpha}}+\triangle S\left(z_{0}\right), \quad z_{0} \in \mathbb{C}, z_{0} \neq z_{\alpha}$.

In particular, in the gauge $S\left(z_{0}\right)=0$, we have $E=0$ and

$$
\frac{\partial S(z)}{\partial z_{\alpha}}=\triangle S(z)
$$

This relation shows a connection of the variations of $z_{\alpha}$ with non isospectral deformations for the dKP hierarchy.

Remark 3.2 Equations (34), (36) and the dKP hierarchy are invariant under the scale transformations

$$
\begin{aligned}
t_{n} & \longrightarrow t_{n}^{\prime}=\lambda t_{n}, \quad n=1,2, \ldots, \quad-\infty<\lambda<\infty \\
F(t) & \rightarrow F^{\prime}\left(t^{\prime}\right)=\lambda^{2} F(t), \\
S(t) & \rightarrow S^{\prime}\left(t^{\prime}\right)=\lambda S(t), \\
u(t) & \rightarrow u^{\prime}\left(t^{\prime}\right)=u(t) .
\end{aligned}
$$

Variation of the function $\mathrm{F}$ due to the infinitesimal variation of the times $t_{n}\left(\delta t_{n}=\varepsilon t_{n}\right)$ is equal to $\delta F=\varepsilon \sum_{n>1} t_{n} \frac{\partial F}{\partial t_{n}}$.

Remark 3.3 Let us introduce the complex Dirichlet type integral

$$
D_{\widetilde{G}}=\frac{1}{2 \pi i} \iint_{\widetilde{G}}\left(\partial S \wedge \bar{\partial} S-\partial S_{0} \wedge \bar{\partial} S_{0}\right)
$$

For the d(KP-mKP) hierarchy one gets, using (20) with $t_{0}=0$, the expansion $\widetilde{S}=\sum_{n \geq 0}\left(z-z_{\alpha}\right)^{n} \widetilde{S}_{n}$ as $z \rightarrow z_{\alpha}$ and the formula (32):

$$
D_{\widetilde{G}}=-\sum_{n \geq 1} n t_{n} \widetilde{S}_{n}=\sum_{n \geq 1} t_{n} \frac{\partial F}{\partial t_{n}} .
$$

Thus, $\delta F=\varepsilon D_{\widetilde{G}}$. An interrelation between the Dirichlet type integrals and $\tau$-functions for dispersionless hierarchies will be discussed in a separate paper. 


\section{Two-points source. Dispersionless Laplace and 2DTL hierarchies}

The case of a source $\rho(h)$ concentrated in two different points $z_{\alpha}$ and $z_{\beta}$ is

rather rich. The domain $\widetilde{G}$ can be a disconnected set $\left(\widetilde{G}=\mathcal{D}_{\alpha} \cup \mathcal{D}_{\beta}\right)$, where $\mathcal{D}_{\alpha}$ and $\mathcal{D}_{\beta}$ are two disks centered at $z_{\alpha}$ and $z_{\beta}$ respectively. Several types of different reduced hierarchies can arise.

The function $S_{0}$ has the form

$$
S_{0}=t_{0 \alpha} \ln \left(z-z_{\alpha}\right)+\sum_{n=1}^{\infty} \frac{x_{n}}{\left(z-z_{\alpha}\right)^{n}}+t_{0 \beta} \ln \left(z-z_{\beta}\right)+\sum_{n=1}^{\infty} \frac{y_{n}}{\left(z-z_{\beta}\right)^{n}} .
$$

Let us first consider variations of the variables $x_{n}$ and $y_{n}$. Similar to the one-point case (26) one obtains two hierarchies of equations

$$
\begin{gathered}
S_{x_{n}}=\sum_{k=0}^{n} U_{k}(x, y)\left(S_{x_{1}}\right)^{k}, \\
S_{y_{n}}=\sum_{k=0}^{n} V_{k}(x, y)\left(S_{y_{1}}\right)^{k}, \quad n \geq 1,
\end{gathered}
$$

where $U_{k}$ and $V_{k}$ are certain functions. Each of these hierarchies gives rise to the dKP-mKP hierarchy in the variables $x_{n}$ and $y_{n}$, respectively. Interconnection between both hierarchies is provided by the equation

$$
S_{x_{1}} S_{y_{1}}+a S_{x_{1}}+b S_{y_{1}}+c=0
$$

where

$$
a=-S_{y_{1}}\left(z_{\alpha}\right), \quad b=-S_{x_{1}}\left(z_{\beta}\right),
$$

and

$$
c=-S_{x_{1}}\left(z_{0}\right) S_{y_{1}}\left(z_{0}\right)-a S_{x_{1}}\left(z_{0}\right)-b S_{y_{1}}\left(z_{0}\right) .
$$

Here $z_{0} \in \mathbb{C}, z_{0} \neq z_{\alpha}, z_{\beta}$.

Equation (55) can be treated as the quasi-classical limit of the well-known Laplace equation

$$
\psi_{\xi \eta}+A \psi_{\xi}+B \psi_{\eta}+c \psi=0
$$


Indeed, by introducing slow variables $x_{1}:=\epsilon \xi, y_{1}:=\epsilon \eta$, considering the quasiclassical wave-function limit $\psi=\exp (S / \epsilon)$ and proceeding to the limit $\epsilon \rightarrow$ 0 , provided that $A\left(\frac{x_{1}}{\epsilon}\right) \rightarrow a\left(x_{1}, y_{1}\right), B\left(\frac{x_{1}}{\epsilon}\right) \rightarrow b\left(x_{1}, y_{1}\right), C\left(\frac{x_{1}}{\epsilon}\right) \rightarrow c\left(x_{1}, y_{1}\right)$, one converts (58) into (55).

So one can refer to the hierarchy described by equations (53)-(55) as the dispersionless Laplace hierarchy. It contains different interesting particular cases. In the gauge $S\left(z_{0}\right)=0$, the lowest member of this hierarchy is given by the equations

$$
\begin{gathered}
S_{x_{1}} S_{y_{1}}+a S_{x_{1}}+b S_{y_{1}}+c=0 \\
S_{x_{2}}=\left(S_{x_{1}}\right)^{2}+U_{1} S_{x_{1}}, \quad S_{y_{2}}=\left(S_{y_{1}}\right)^{2}+V_{1} S_{y_{1}}
\end{gathered}
$$

where $U_{1}=-2 \widetilde{S}_{x_{1}}\left(z_{\alpha}\right), V_{1}=-2 \widetilde{S}_{y_{1}}\left(z_{\beta}\right)$. Evaluating equations (59) at $z=$ $z_{\alpha}, z_{\beta}$, one gets the following equations

$$
\begin{aligned}
& U_{x_{2}}=\frac{2}{z_{\beta}-z_{\alpha}}\left(U_{x_{1}}-V_{x_{1}}\right)+\left(U_{x_{1}}\right)^{2}-2 U_{x_{1}} V_{x_{1}}, V_{x_{2}}+\left(V_{x_{1}}\right)^{2}=2 \phi_{x_{1}}, \\
& U_{x_{1}} V_{y_{1}}-\frac{1}{z_{\alpha}-z_{\beta}} V_{y_{1}}+\frac{1}{z_{\alpha}-z_{\beta}} U_{x_{1}}+\phi_{y_{1}}=0, \\
& V_{y_{2}}=\frac{2}{\left(z_{\alpha}-z_{\beta}\right)}\left(V_{y_{1}}-U_{y_{1}}\right)+\left(V_{y_{1}}\right)^{2}-2 V_{y_{1}} U_{y_{1}}, \\
& U_{y_{2}+\left(U_{y_{1}}\right)^{2}}=2 \widetilde{\varphi}_{y_{1}}, \\
& \widetilde{\varphi}_{x_{1}}=\varphi_{y_{1}}
\end{aligned}
$$

where $\quad U=\widetilde{S}\left(z_{\beta}\right), V=\widetilde{S}\left(z_{\alpha}\right), \phi=\left.\frac{\partial \widetilde{S}}{\partial z}\right|_{z=z_{\alpha}}, \widetilde{\phi}=\left.\frac{\partial \widetilde{S}}{\partial z}\right|_{z=z_{\beta}}$.

Equations (59) and (60) are the lowest members of the dispersionless Laplace hierarchy. In terms of the variable $t:=x_{2}+y_{2}$, equations (60) are equivalent to the dispersionless limit of the system of equations considered in [38] (equation (9.6)) and in [39]. The equations discussed in [39] are the equations for the Davey-Stewartson wave function. One more case of interesting application of the dispersionless Laplace transform corresponds to the constraints $S\left(z_{\alpha}\right)=S\left(z_{\beta}\right)=$ const. This constraint is compatible with equations (53) and (54) for odd $n$. The lowest member of such a hierarchy 
is given by the equations

$$
\begin{gathered}
S_{x_{1}} S_{y_{1}}+q=0, \\
S_{x_{3}}=\left(S_{x_{1}}\right)^{3}+U_{1} S_{x_{1}}, \\
S_{y_{3}}=\left(S_{y_{1}}\right)^{3}+V_{1} S_{x_{1}},
\end{gathered}
$$

where $U_{1}=3 \partial_{y_{1}}^{-1} q_{x_{1}}, V_{1}=3 \partial_{x_{1}}^{-1} q_{y_{1}}$. The corresponding equations for the potential $q$ are

$$
q_{x_{3}}=3\left(q\left(\partial_{y_{1}}^{-1} q_{x_{1}}\right)\right)_{x_{1}}, \quad q_{y_{3}}=3\left(q\left(\partial_{x_{1}}^{-1} q_{y_{1}}\right)\right)_{y_{1}} .
$$

In terms of $\partial_{t}=\partial_{x_{3}}+\partial_{y_{3}}$ one has

$$
q_{t}=3\left(q\left(\partial_{y_{1}}^{-1} q_{x_{1}}\right)\right)_{x_{1}}+3\left(q\left(\partial_{x_{1}}^{-1} q_{y_{1}}\right)\right)_{y_{1}} .
$$

Equation (63) is the dispersionless limit of the Nizhnik-Veselov-Novikov equation [40-41]

$$
q_{t}=q_{x_{1} x_{1} x_{1}}+q_{y_{1} y_{1} y_{1}}+3\left(q\left(\partial_{y_{1}}^{-1} q_{x_{1}}\right)_{x_{1}}+3\left(q\left(\partial_{x_{1}}^{-1} q_{y_{1}}\right)_{y_{1}} .\right.\right.
$$

Notice that the dispersionless limit of the Veselov-Novikov equation was discussed for the first time in [4].

Equations (61) have an interesting application in physics. Namely, the first equation is, in fact, the eiconal equation from the geometric optics. Indeed, if we denote $z=x_{1}, \bar{z}=y_{1}, z=x+i y$, it reads

$$
S_{x}^{2}+S_{y}^{2}=-4 q .
$$

Remark 4.1 For the multipoint sources case, the gauge $S\left(z_{0}\right)=$ const., $z_{0} \neq$ $z_{\alpha}, z_{\beta}$ is a symmetric and natural one.

Remark 4.2 Equation (55) implies that

$$
S_{y_{1}}=-\frac{a S_{x_{1}}+c}{S_{x_{1}}+b} .
$$

This relation shows that, using only one source $z_{\alpha}$, one can generate infinitesimal deformations of $S$ which have poles at other points (in this case at $z=z_{\beta}$ ). This fact, which was already discussed in Section 2, means that, in particular, it is convenient to introduce the corresponding times from the very beginning. 
Let us include now the logarithmic times into consideration. Analogously to (40), we have in the gauge $S\left(z_{0}\right)=0$

$$
\begin{gathered}
S_{x_{n}}=\sum_{k=0}^{n} A_{k} e^{-k S_{t_{0 \alpha}},} \\
S_{y_{n}}=\sum_{k=0}^{n} B_{k} e^{-k S_{t_{0 \beta}}}, \quad n \geq 1,
\end{gathered}
$$

where $A_{k}$ and $B_{k}$ are functions depending on the whole set of times. In addition there is an equation relating the derivatives of $S$ with respect to the times $t_{0 \alpha}$ and $t_{0 \beta}$

$$
e^{-S_{t_{0 \alpha}}-S_{t_{0 \beta}}}+\widetilde{A} e^{-S_{t_{0 \alpha}}}+\widetilde{B} e^{-S_{t_{0 \beta}}}+\widetilde{C}=0
$$

where

$$
\begin{gathered}
\widetilde{A}=\frac{1}{z_{\beta}-z_{\alpha}} e^{-S_{t_{0 \beta}}\left(z_{\alpha}\right)}, \\
\widetilde{B}=\frac{1}{z_{\alpha}-z_{\beta}} e^{-S_{t_{0 \alpha}}\left(z_{\beta}\right)}, \quad \widetilde{C}=-1-\widetilde{A}-\widetilde{B} .
\end{gathered}
$$

Equations (65)-(66) describe the general two-points hierarchy of the infinitesimal deformations. It contains a well-known dispersionless integrable hierarchy. Indeed, let us introduce new variables $T$ and $t$ such that $t_{0 \alpha}=$ $T+t, t_{0 \alpha}=T-t$. Then one has

$$
e^{ \pm S_{t}}=\left(\frac{z-z_{\alpha}}{z-z_{\beta}}\right)^{ \pm 1} e^{ \pm \widetilde{S}_{t}}
$$

Using (68), one obtains

$$
S_{x_{n}}=\sum_{k=0}^{n} U_{k} e^{-k S_{t}}, \quad S_{y_{n}}=\sum_{k=0}^{n} V_{k} e^{k S_{t}}, \quad n \geq 1,
$$

for certain functions $U_{k}$ and $V_{k}$. The lowest member of the hierarchy (69) is

$$
S_{x_{1}}=U e^{-S_{t}}-U, \quad S_{y_{1}}=V e^{S_{t}}-V
$$


where

$$
U=\frac{1}{z_{\alpha}-z_{\beta}} e^{\widetilde{S}_{t}\left(z_{\alpha}\right)}, \quad V=\frac{1}{z_{\beta}-z_{\alpha}} e^{-\widetilde{S}_{t}\left(z_{\beta}\right)}
$$

Evaluating the left-hand sides of the equations $(70)$ at $z=z_{\beta}$ and $z_{\alpha}$, one gets

$$
\phi_{x_{1}}=\frac{1}{z_{\alpha}-z_{\beta}}\left(1-e^{\psi_{t}}\right), \quad \psi_{y_{1}}=\frac{1}{z_{\beta}-z_{\alpha}}\left(1-e^{-\phi_{t}}\right)
$$

where $\phi=\widetilde{S}\left(z_{\beta}\right), \psi=\widetilde{S}\left(z_{\alpha}\right)$, or

$$
U_{y_{1}}+U V_{t}=0, \quad V_{x_{1}}-V U_{t}=0 .
$$

The system (71) implies

$$
\theta_{x_{1} y_{1}}+\left(e^{\theta}\right)_{t t}=0
$$

where

$$
\theta=\ln \left[-\frac{1}{\left(z_{\alpha}-z_{\beta}\right)^{2}} e^{\psi_{t}-\phi_{t}}\right]=\lg (U V)
$$

Equation $(73)$ is the well-known dispersionless 2DTL equation (see [11]). Its associated system of Hamilton-Jacobi equations is provided by (69) [11, 21].

Note that equations (70) imply the following equation for $S$

$$
S_{x_{1} y_{1}}+S_{x_{1}} S_{y_{1}}\left(\frac{1}{e^{S_{t}}-1}\right)_{t}=0
$$

Now we will derive generating and addition formulae for the dispersionless 2DTL hierarchy in the gauge $S\left(z_{0}\right)=0$. To this end we introduce the operators

$$
\mathcal{D}_{\alpha}(z):=\sum_{n=1}^{\infty} \frac{1}{n}\left(z-z_{\alpha}\right)^{n} \frac{\partial}{\partial x_{n}}, \quad \mathcal{D}_{\beta}(z):=\sum_{n=1}^{\infty} \frac{1}{n}\left(z-z_{\beta}\right)^{n} \frac{\partial}{\partial y_{n}} .
$$

One has

$$
\begin{aligned}
& e^{-\mathcal{D}_{\alpha}\left(z_{1}\right) S(z)}=\frac{z-z_{1}}{z-z_{\alpha}} e^{-\mathcal{D}_{\alpha}\left(z_{1}\right) \widetilde{S}(z)}, \\
& e^{-\mathcal{D}_{\beta}\left(z_{2}\right) S(z)}=\frac{z-z_{2}}{z-z_{\beta}} e^{-\mathcal{D}_{\beta}\left(z_{2}\right) \widetilde{S}(z)},
\end{aligned}
$$


where $z \in \mathbb{C}, z_{1}, z_{2} \in \widetilde{G}$. Using $(77)$ one obtains for $p_{\alpha}:=\frac{\partial S}{\partial x_{1}}$ and $p_{\beta}:=\frac{\partial S}{\partial y_{1}}$

$$
\begin{aligned}
& p_{\alpha}(z)-p_{\alpha}\left(z_{1}\right)+\frac{1}{z_{1}-z_{\alpha}} e^{-\mathcal{D}_{\alpha}\left(z_{1}\right)\left(S(z)-\widetilde{S}\left(z_{\alpha}\right)\right)}=0, z \in \mathbb{C}, z_{1} \in \widetilde{G}, \\
& p_{\beta}(z)-p_{\beta}\left(z_{2}\right)+\frac{1}{z_{2}-z_{\beta}} e^{-\mathcal{D}_{\beta}\left(z_{2}\right)\left(S(z)-\widetilde{S}\left(z_{\beta}\right)\right)}=0, z \in \mathbb{C}, z_{2} \in \widetilde{G} .
\end{aligned}
$$

By proceeding as in the one-point case, one finds that equations (78) imply that

$$
\widetilde{S}(z)=\widetilde{S}\left(z_{\alpha}\right)-\mathcal{D}_{\alpha}(z) F_{\alpha}, \quad \widetilde{S}(z)=\widetilde{S}\left(z_{\beta}\right)-\mathcal{D}_{\beta}(z) F_{\beta}, z \in \widetilde{G},
$$

where $F_{\alpha}$ and $F_{\beta}$ are functions depending on the times only, which satisfy addition formulae of the form (34).

On the other hand, using (68) one gets the identities

$$
\begin{aligned}
& p_{\alpha}(z)-p_{\alpha}\left(z_{\beta}\right)=\frac{1}{z_{\alpha}-z_{\beta}} e^{-D\left(S(z)-\widetilde{S}\left(z_{\alpha}\right)\right)}, \\
& p_{\beta}(z)-p_{\beta}\left(z_{\alpha}\right)=\frac{1}{z_{\beta}-z_{\alpha}} e^{-D\left(S(z)-\widetilde{S}\left(z_{\beta}\right)\right)},
\end{aligned}
$$

where $z \in \mathbb{C}$ and we denote $D:=\frac{\partial}{\partial t}$. By substituting (80) into (78), we obtain

$$
\begin{aligned}
& \frac{1}{z_{\alpha}-z_{\beta}} e^{-D\left(S(z)-\widetilde{S}\left(z_{\alpha}\right)\right)}-\frac{1}{z_{\alpha}-z_{\beta}} e^{-D\left(S\left(z_{1}\right)-\widetilde{S}\left(z_{\alpha}\right)\right)} \\
& \quad+\frac{1}{z_{1}-z_{\alpha}} e^{-\mathcal{D}_{\alpha}\left(z_{1}\right)\left(S(z)-\widetilde{S}\left(z_{\alpha}\right)\right)}=0, \quad z \in \mathbb{C}, z_{1} \in \widetilde{G}
\end{aligned}
$$

and

$$
\begin{aligned}
& \frac{1}{z_{\beta}-z_{\alpha}} e^{-D\left(S(z)-\widetilde{S}\left(z_{\beta}\right)\right)}-\frac{1}{z_{\beta}-z_{\alpha}} e^{-D\left(S\left(z_{2}\right)-\widetilde{S}\left(z_{\beta}\right)\right)} \\
& \quad+\frac{1}{z_{2}-z_{\beta}} e^{-\mathcal{D}_{\beta}\left(z_{2}\right)\left(S(z)-\widetilde{S}\left(z_{\beta}\right)\right)}=0, \quad z \in \mathbb{C}, z_{2} \in \widetilde{G} .
\end{aligned}
$$

These identities are generating equations for (69). Indeed, expanding their left-hand sides in Taylor series in $z_{1}$ and $z_{2}$, one gets (69). Furthermore, 
evaluating (81) at $z=z_{\beta}$ and $z=z_{\alpha}$, one deduces that $\widetilde{S}\left(z_{\alpha}\right)-\widetilde{S}\left(z_{\beta}\right)=$ $D F_{\alpha}=D F_{\beta}$ and so on. Hence

$$
F_{\alpha}=F_{\beta}=F=\ln \tau \mathrm{d} 2 \mathrm{DTL} .
$$

Thus there is only one $\tau$-function for the d2DTL hierarchy. This feature is in agreement with earlier results [11]. Considering (81) at $z=\tilde{z}_{1} \in \widetilde{G}$ and $z=\tilde{z}_{2} \in \widetilde{G}$, and using (82), one shows that the function $F$ satisfies

$$
\begin{gathered}
\left(\tilde{z}_{1}-z_{\beta}\right)\left(z_{1}-z_{\alpha}\right) e^{D \mathcal{D}_{\alpha}\left(\tilde{z}_{1}\right) F}-\left(\tilde{z}_{1}-z_{\beta}\right)\left(\tilde{z}_{1}-z_{\alpha}\right) e^{D \mathcal{D}_{\alpha}\left(z_{1}\right) F}+ \\
+\left(z_{\alpha}-z_{\beta}\right)\left(\tilde{z}_{1}-z_{1}\right) e^{\mathcal{D}_{\alpha}\left(z_{1}\right) \mathcal{D}_{\alpha}\left(\tilde{z}_{1}\right) F}=0
\end{gathered}
$$

and

$$
\begin{gathered}
\left(\tilde{z}_{2}-z_{\alpha}\right)\left(z_{2}-z_{\beta}\right) e^{-D \mathcal{D}_{\beta}\left(\tilde{z}_{2}\right) F}-\left(\tilde{z}_{2}-z_{\alpha}\right)\left(\tilde{z}_{2}-z_{\beta}\right) e^{-D \mathcal{D}_{\alpha}\left(z_{2}\right) F}+ \\
+\left(z_{\beta}-z_{\alpha}\right)\left(\tilde{z}_{2}-z_{2}\right) e^{\mathcal{D}_{\beta}\left(z_{2}\right) \mathcal{D}_{\alpha}\left(\tilde{z}_{2}\right) F}=0 .
\end{gathered}
$$

These identities imply, in particular, the addition formulae for the dKP hierarchy.

Then, evaluating (81) at $z=z_{2}$, using the relations $\widetilde{S}\left(z_{2}\right)=\widetilde{S}\left(z_{\beta}\right)-$ $\mathcal{D}_{\beta}\left(z_{2}\right) F$ and $\widetilde{S}\left(z_{\alpha}\right)-\widetilde{S}\left(z_{\beta}\right)=D F$, one obtains

$$
\begin{aligned}
1 & +\frac{\left(z_{\alpha}-z_{\beta}\right)\left(z_{1}-z_{\alpha}\right)}{\left(z_{1}-z_{\beta}\right)\left(z_{2}-z_{\alpha}\right)} e^{\mathcal{D}_{\alpha}\left(z_{1}\right) \mathcal{D}_{\beta}\left(z_{2}\right) F} \\
& -\frac{\left(z_{1}-z_{\alpha}\right)\left(z_{2}-z_{\beta}\right)}{\left(z_{1}-z_{\beta}\right)\left(z_{2}-z_{\alpha}\right)} e^{\left[\mathcal{D}_{\beta}\left(z_{2}\right)-\mathcal{D}_{\alpha}\left(z_{1}\right)+D\right] D F}=0 .
\end{aligned}
$$

Evaluating equation (82) at $z=z_{1}$ and taking into account that $\widetilde{S}\left(z_{1}\right)=$ $\widetilde{S}\left(z_{\alpha}\right)-\mathcal{D}_{\alpha}\left(z_{1}\right) F$, one gets the same equation (86).

Equations (84), (85) and (86) form a complete set of addition formulae for the d2DTL hierarchy which completely define [11, [18] its associated $\tau$ function.

Similarly to the one-point case, one can derive a generating equation for the hierarchy of equations for $S$ (see [37])

Finally, let us include into consideration the variations of positions $z_{\alpha}$ and $z_{\beta}$ of singularities. The simplest case is given by the one logarithmic source

$$
S_{0}=t \ln \frac{z-z_{\alpha}}{z-z_{\beta}}
$$


Since

$$
S_{z_{\alpha}}=-\frac{t}{z-z_{\alpha}}+\frac{\partial \widetilde{S}}{\partial z_{\alpha}}, \quad S_{z_{\beta}}=\frac{t}{z-z_{\beta}}+\frac{\partial \widetilde{S}}{\partial z_{\beta}},
$$

one obtains the following equations (we use the gauge $S\left(z_{0}\right)=0$ )

$$
S_{z_{\alpha}}=U e^{-S_{t}}-U, \quad S_{z_{\beta}}=V e^{S_{t}}-V
$$

where

$$
U=\frac{t}{z_{\alpha}-z_{\beta}} e^{\widetilde{S}_{t}\left(z_{\alpha}\right)}, \quad V=\frac{t}{z_{\beta}-z_{\alpha}} e^{-\widetilde{S}_{t}\left(z_{\beta}\right)} .
$$

Equations (89) imply that $\theta=\lg (U V)$ obeys

$$
\theta_{z_{\alpha} z_{\beta}}+\left(e^{\theta}\right)_{t t}=0
$$

which is again the d2DTL equation (73), but now the spacial variables are the positions of the logarithmic singularities. In particular for $z_{\alpha}=w, z_{\beta}=\bar{w}$ one gets the elliptic version of the d2DTL hierarchy. Despite of the fact that (91) coincides with (73) their corresponding dressing procedures are different.

Indeed, for the simplest choice $\widetilde{S}\left(z_{\alpha}\right):=\psi=0, \widetilde{S}\left(z_{\beta}\right):=\phi=0$, the formula (75) gives the trivial solution $\theta=$ const. of (73), while for (91) one gets the nontrivial solution

$$
\theta=\lg (U V)=2 \lg \left(\frac{t}{z_{\beta}-z_{\alpha}}\right) .
$$

For the whole d2DTL hierarchy one has two relations of the type (50).

\section{Multi-point sources}

For the general $N$ points-like source

$$
S_{0}=\sum_{\alpha=1}^{N} t_{0 \alpha} \lg \left(z-z_{\alpha}\right)+\sum_{\alpha=1}^{N} \sum_{n=1}^{\infty} \frac{t_{n \alpha}}{\left(z-z_{\alpha}\right)^{n}},
$$

one has $N$ hierarchies of equations

$$
S_{t_{n \alpha}}=\sum_{k=0}^{n} U_{\alpha k} e^{-k S_{t_{0 \alpha}}}, \quad \alpha=1, \ldots, N, n \geq 1
$$


These families of equations are related by equations of the type (66)

$$
e^{-S_{t_{0 \alpha}}-S_{t_{0 \beta}}}+A_{\alpha \beta} e^{-S_{t_{0 \alpha}}}+A_{\beta \alpha} e^{-S_{t_{0 \beta}}}+B_{\alpha \beta}=0,
$$

where

$$
A_{\alpha \beta}=e^{-S_{t_{0 \beta}}\left(z_{\alpha}\right)}, \quad B_{\alpha \beta}=-\left(1+A_{\alpha \beta}+A_{\beta \alpha}\right) .
$$

The hierarchy of equations (93), (94) is nothing but the universal Whitham hierarchy introduced in [8]. It contains a number of interesting subhierarchies. Obviously, there are $N \mathrm{~d}(\mathrm{KP}-\mathrm{mKP})$ hierarchies associated with each of the singularity points $z_{\alpha}(\alpha=1, \ldots, N)$. Then there are d2DTL hierarchies corresponding to each pair $\left(z_{\alpha}, z_{\beta}\right)$ of the singularity points. One can show that there is only one function $F$ for (93), (94) [8]. To demonstrate this property it is enough to apply the same argumentation as that used in the previous section to identify each pair $F_{\alpha}$ and $F_{\beta}$.

We will discuss now some of the subhierarchies of (93), (94) with more that two singularity points. Firstly, by restricting ourselves to the variables $t_{1 \alpha}(\alpha=1, \ldots, N)$, we obtain the following set of equations (in the gauge $\left.S\left(z_{0}\right)=0\right)$

$$
S_{\xi_{\alpha}} S_{\xi_{\beta}}-\varphi_{\xi_{\beta}}^{\alpha} S_{\xi_{\alpha}}-\varphi_{\xi_{\alpha}}^{\beta} S_{\xi_{\beta}}=0, \quad \alpha \neq \beta, \alpha, \beta=1, \ldots, N,
$$

where $\xi_{\alpha}:=t_{1 \alpha}$ and $\varphi^{\alpha}:=S\left(z_{\alpha}\right)$. By evaluating the left-hand side of (96) at the points $z=z_{\gamma}(\gamma \neq \alpha, \beta)$ one gets the bilinear system of equations

$$
\varphi_{\xi_{\alpha}}^{\gamma} \varphi_{\xi_{\beta}}^{\gamma}-\varphi_{\xi_{\beta}}^{\alpha} \varphi_{\xi_{\alpha}}^{\gamma}-\varphi_{\xi_{\alpha}}^{\beta} \varphi_{\xi_{\beta}}^{\gamma}=0
$$

where $\alpha, \beta$ and $\gamma$ are different. Equivalently

$$
\frac{\varphi_{\xi_{\beta}}^{\alpha}}{\varphi_{\xi_{\beta}}^{\gamma}}+\frac{\varphi_{\xi_{\alpha}}^{\beta}}{\varphi_{\xi_{\alpha}}^{\gamma}}=1
$$

Equations (96) and (97) can be treated as the dispersionless limits of the systems

$$
\begin{gathered}
\psi_{u_{\alpha} u_{\beta}}=\left(\lg H^{\alpha}\right)_{u_{\beta}} \psi_{u_{\alpha}}+\left(\lg H^{\beta}\right)_{u_{\alpha}} \psi_{u_{\beta}}, \\
H_{u_{\alpha} u_{\beta}}^{\gamma}=\left(\lg H^{\alpha}\right)_{u_{\beta}} H_{u_{\alpha}}^{\gamma}+\left(\lg H^{\beta}\right)_{u_{\alpha}} H_{u_{\beta}}^{\gamma},
\end{gathered}
$$


where $\alpha, \beta$ and $\gamma$ are different. The equations (100) form the Darboux system describing the $N$-conjugate systems of surfaces in Euclidean space, and (99) are the corresponding equations for the position vector [42]. In this case the slow variables are $\xi_{\alpha}=\epsilon u_{\alpha}$ and the quasiclassical limit is implemented by the expressions $\psi=\exp \frac{S}{\epsilon}, H^{\alpha}=\exp \frac{\varphi^{\alpha}}{\epsilon}$. Thus, one shows that (99) anbd (100) represent the dispersionless limit of the Darboux system and its associated linear system (see also 37]).

Our second example is determined by an $S_{0}$ function of the form

$$
S_{0}=\frac{x}{z-z_{0}}+y \sum_{n=1}^{N} \frac{a_{n}}{z-z_{n}}+t \sum_{m=1}^{M} \frac{b_{m}}{z-\tilde{z}_{m}},
$$

where $a_{n}, b_{m}$ are arbitrary complex parameters and the sets $\left\{z_{n}\right\}$ and $\left\{\tilde{z}_{m}\right\}$ are disjoint. In this case

$$
\begin{gathered}
p:=S_{x}=\frac{1}{z-z_{0}}+\widetilde{S}_{x}, \\
S_{y}=\sum_{n=1}^{N} \frac{a_{n}}{z-z_{n}}+\widetilde{S}_{y}, \\
S_{t}=\sum_{m=1}^{M} \frac{b_{m}}{z-\tilde{z}_{m}}+\widetilde{S}_{t} .
\end{gathered}
$$

From these expressions the following Hamilton-Jacobi type equations follow

$$
S_{y}=V_{0}+\sum_{n=1}^{N} \frac{V_{n}}{S_{x}-U_{n}}, \quad S_{t}=\widetilde{V}_{0}+\sum_{m=1}^{M} \frac{\widetilde{V}_{m}}{S_{x}-\widetilde{U}_{m}},
$$

where

$$
U_{n}=S_{x}\left(z_{n}, x, y, t\right), \quad \widetilde{U}_{m}=S_{x}\left(\tilde{z}_{m}, x, y, t\right),
$$

and $V_{0}, V_{n}, \widetilde{V}_{0}, \widetilde{V}_{m}$ are functions of $(x, y, t)$. The type of equations (103) as well as its associated integrable systems have been considered in [10].

Another interesting subhierarchy corresponds to the choice

$$
S_{0}=\frac{x}{z-z_{0}}+\frac{t}{\left(z-z_{0}\right)^{2}}+y \sum_{n=1}^{N} \frac{a_{n}}{z-z_{n}} .
$$


In this case one has the following equations

$$
S_{y}=V_{0}+\sum_{n=1}^{N} \frac{V_{n}}{S_{x}-U_{n}}, \quad S_{t}=S_{x}^{2}+U_{0},
$$

where $U_{n}:=S_{x}\left(z_{n}, x, y, t\right)$. The associated integrable system

$$
\begin{gathered}
U_{n, t}+U_{0, x}-\left(U_{n}^{2}\right)_{x}=0, \\
V_{n, t}-2\left(V_{n} U_{n}\right)_{x}=0, \quad 1 \leq n \leq N, \\
U_{0, y}+2 \sum_{i=1}^{N} V_{n, x}=0,
\end{gathered}
$$

is the generalization of the Benney system to $2+1$ dimensions proposed in [8], [10]

Other particular cases of the hierarchy (93),(94) will be considered elsewhere.

Remark 5.1 All of our constructions in this and previous sections were local ones, with singularities (sources) located in certain domains of $\mathbb{C}$. This means that one will get the same formulae by considering instead a chart of the Riemann sphere. Compactness of the Riemann sphere imposes certain constraints of the form (92) of the sources (singularities). In particular, $\sum_{\alpha=1}^{N} t_{0 \alpha}=0$ (see e.g. 34 $)$.

Acknowledgements The authors are very grateful to the Isaac Newton Institute for Mathematical Sciences of Cambridge, where this work has been done and written, for the kind hospitality. They are also grateful to the organizers of the programme "Integrable Systems" for the support provided. L. Martinez Alonso wish to thank the Fundación Banco Bilbao Vizcaya Argentaria for supporting his stay at Cambridge University as a BBV visiting professor.

\section{References}

[1] B. A. Kupershmidt and Yu. I. Manin, Long wave equations with a free boundary, I , Funkts. Anal. Prilozh. 11(3): 31 (1977); II, Funkts .Anal. Prilozh. 12 (1): 25 (1978); D.R. Lebedev and Yu.I. Manin, Conservation laws and Lax representation of Benney's long wave equations, Phys. Lett. 74A : 154-156 (1979). 
[2] V. E. Zakharov, Benney equations and quasi-classical approximation in the inverse problem method, Func. Anal. Priloz. 14: 89-98 (1980).

[3] P. D. Lax and C. D. Levermore, The small dispersion limit on the Korteweg-de Vries equation, Commun. Pure Appl. Math. 36: 253-290, 571-593, 809-830 (1983).

[4] I. M. Krichever, Averaging method for two-dimensional integrable equations, Funkts . Anal. Priloz. 22: 37-52 (1988).

[5] Y. Kodama, A method for solving the dispersionless KP equation and its exact solutions, Phys. Lett. 129A: 223-226 (1988); Y. Kodama, Solutions of the dispersionless Toda equation, Phys.Lett. 147A: 477-482 (1990).

[6] B. A. Dubrovin and S. P. Novikov, Hydrodynamics of weakly deformed soliton lattices: differential geometry and Hamiltonian theory, Russian Math. Surveys, 44: 35-124 (1989).

[7] K. Takasaki and T. Takebe, $\operatorname{SDIFF}(2) \mathrm{KP}$ hierarchy, Int. J. Mod. Phys. A, Vol.7, Suppl.1B: 889-922 (1992).

[8] I. M. Krichever, The $\tau$-function of the universal Whitham hierarchy, matrix models and topological field theories, Commun. Pure Appl. Math. 47: 437-475 (1994).

[9] Singular limits of dispersive waves (eds. N. M. Ercolani et al), Nato Adv. Sci. Inst. Ser. B Phys. 320 , Plenum, New York (1994).

[10] V. E. Zakharov, Dispersionless limit of integrable system in $2+1$ dimensions, in [9], pp 165-174 (1994).

[11] K. Takasaki and T. Takebe, Integrable hierarchies and dispersionless limit, Rev. Math. Phys. 7: 743-818 (1995).

[12] R. Carroll and Y. Kodama, Solutions of the dispersionless Hirota equations, J. Phys. A Math. Gen. 28: 6373-6387 (1995).

[13] S. Jin, C. D. Levermore and D. W. McLaughlin, The semiclassical limit of the defocusing NLS hierarchy, Comm. Pure and Appl. Math. 52: 613-654 (1999). 
[14] I. M. Krichever, The dispersionless Lax equations and topological minimal models, Commun. Math. Phys. 143: 415-429 (1992).

[15] B. A. Dubrovin, Hamiltonian formalism of Whitham-type hierarchies and topological Landau-Ginzburg models, Commun. Math. Phys. 145: 195-203 (1992); B. A. Dubrovin and Y. Zhang, Bihamiltonian hierarchies in 2D topological field theory at one-loop approximation, Commun. Math. Phys. 198: 311-361 (1998); B.A.Dubrovin and Y.Zhang, Normal forms of hierarchies of integrable PDEs, Frobenious manifolds and Gromov-Witten invariants, arXiv:math.DG/0108160 v1 (2001).

[16] S. Aoyama and Y. Kodama, Topological Landau-Ginzburg theory with rational potential and the dispersionless KP hierarchy, Commun.Math.Phys. 182: 185-219 (1996) ; M. Dunaiski, L.J. Mason and P. Tod, Einstein-Weyl geometry, the dKP equation and twistor theory, J.Geom.Phys. 37: 63-93 (2001); L.A. Takhtajan, Free bosons and taufunction for compact Riemann surfaces and smooth Jordan curves I. Current correlation functions, Lett.Math.Phys. 56 : 181-228 (2001).

[17] J. Gibbons and S. P. Tsarev, Conformal maps and reductions of the Benney equations, Phys. Lett. 258A: 263-271 (1999).

[18] P. B. Wiegmann and A. Zabrodin, Conformal maps and integrable hierarchies, Commun. Math. Phys. 213: 523-538 (2000); M. MineevWeinstein, P. B. Wiegmann and A. Zabrodin, Integrable structure of interface dynamics, Phys. Rev. Lett. 84: 5106-5108 (2000) ; I.K. Kostov, I.Krichever, M. Mineev-Weinstein, P.B. Wiegmann and A. Zabrodin, $\tau$ function for analytic curves, in Random matrices and their applications , MSRI Publications, v. 40, (2001), pp 1-15; A. Marshakov, P. Wiegmann and A. Zabrodin, Integrable structure of the Dirichlet boundary problem in two dimensions, arXiv:hep-th/0109048 (2001).

[19] B. G. Konopelchenko, L. Martinez Alonso and O. Ragnisco, The $\bar{\partial}-$ approach to the dispersionless KP hierarchy, J. Phys. A: Math. Gen. 34: 10209-10217 (2001).

[20] B. G. Konopelchenko and L. Martinez Alonso, $\bar{\partial}$ - equations, integrable deformations of quasiconformal mappings and Whitham hierarchy, Phys. Lett. 286A: 161-166 (2001). 
[21] B. G. Konopelchenko and L. Martinez Alonso, Dispersionless scalar hierarchies, Whitham hierarchy and the quasi-classical $\bar{\partial}$-method, arXiv: nlin: SI/0105071 (2001) .

[22] L. V. Ahlfors, Lectures on quasi-conformal mappings, D. Van Nostrand C. , Princeton, 1966.

[23] Q. Lehto and K. I. Virtanen, Quasiconformal mappings in the plane, Springer-Verlag, Berlin, 1973.

[24] S. L. Krushkal, Quasiconformal mappings and Riemann surfaces, John Wiley and Sons, New-York, 1979.

[25] R. P. Gilbert and Wen Guo-Chun, Free boundary problems occuring in planar fluid dynamics, Nonlinear Anal., Theory, Methods, Appl. 13: 285-303 (1989).

[26] M. Boukrouche, The quasiconformal mapping methods to solve a free boundary problem for generalized Hele-Show flows, Complex Variables 28: 227-242 (1996).

[27] B. Bojarski, Quasiconformal mappings and general structural properties of systems of nonlinear equations elliptic in the sense of Lavrent'ev , Symposia Mathematica XVIII: 485-499 (1976).

[28] T. Iwaniec, Quasiconformal mapping problem for general nonlinear systems of partial differential equations , Symposia Mathematica XVIII: 501-517 (1976).

[29] O. Martio, Partial differential equations and quasiregular mappings, in Lecture Notes in Math. Vol 1508, pp 65-79 (1992)

[30] V. E. Zakharov and S. V. Manakov, Construction of multidimensional nonlinear integrable systems and their solutions, Funkts. Anal. Prilozh. 19: 89-101 (1985).

[31] V. E. Zakharov, On the inverse method, in Inverse problems in action (P. Sabatier, Ed.) p.602, Springer-Verlag, Berlin, 1990.

[32] B. G. Konopelchenko, Solitons in multidimensions, World Scientific, Singapore, 1993. 
[33] I. N. Vekua, Generalized analytic functions, Pergamon Press, Oxford, 1962.

[34] A. Hurwitz and R. Courant , Theory of functions, Springer-Verlag, Berlin, 1964; G. Springer, Introduction to Riemann surfaces, Chelsea P.C., New York, 1981.

[35] T. Miwa, On Hirota's difference equations, Proc. Japan Acad. 58 Ser.A : 8-11 (1982).

[36] B. A. Kuperschmidt, The quasiclassical limit of the modified KP hierarchy, J. Phys. A: Math. Gen. 23: 876-886 (1990).

[37] L. V. Bogdanov, B. Konopelchenko and L. Martinez Alonso, Quasiclassical $\bar{\partial}$-dressing method: generating equations for dispersionless integrable hierarchies, arXiv:nlin.SI/0111062 (2001).

[38] B. G. Konopelchenko, Soliton eigenfunction equations: the IST integrability and some properties, Rev. Math. Phys. 2: 399-440 (1990).

[39] B. G. Konopelchenko, Nets in $R^{3}$, their integrable evolutions and the DS hierarchy, Phys. Lett. 183A: 153-159 (1993).

[40] L. P. Nizhnik, Integration of multidimensional nonlinear equations by the method of inverse problem, DAN SSSR 254: 332 (1980).

[41] A. P. Veselov and S. P. Novikov, Finite-zone two-dimensional potential Schrodinger operators. Explicit formulae and evolution equations, DAN SSSR 279: 20-24 (1984).

[42] G. Darboux, Lecons sur les systemes orthogonaux et les coordonnes curvilignes, Hermann, Paris, 1910. 